\title{
Infectious Mononucleosis and Lyme Disease as Confounding Diagnoses: A Report of 2 Cases
}

\author{
Trever M. Koester, BS; Jennifer K. Meece, PhD; Thomas R. Fritsche, MD; and \\ Holly M. Frost, MD
}

\begin{abstract}
Lyme disease and infectious mononucleosis are common illnesses that share similar clinical presentations. Significant cross-reactivity is known to occur between Lyme and EBV serologic assays complicating the diagnosis. To date, no prior cases of concurrent acute Lyme and EBV infections have been reported. We describe the clinical presentation of two children with confirmed early Lyme disease and features suggestive of infectious mononucleosis, including one case of probable Lyme and EBV co-infection.
\end{abstract}

Keywords: Lyme disease; Epstein-Barr virus; Infectious mononucleosis; Borrelia burgdorferi; Co-infection

$\mathrm{L}$ yme disease and infectious mononucleosis are common illnesses that share similar clinical presentations that are predominantly composed of non-specific symptoms including fatigue, fever, myalgia, arthritis, headaches, neck soreness, and lymphadenopathy. Both are common childhood illnesses in Lyme endemic regions. ${ }^{1}$ Up to twenty-five percent of children with Lyme disease do not present with erythema migrans, further complicating the diagnosis. ${ }^{2}$ Thus, it is common for both Lyme and EBV serologic or heterophile antibody tests to be ordered for children who present with a fever of unknown origin and fatigue in Lyme endemic regions. Significant cross-reactivity is known to occur between Lyme and EBV serologic assays and prior case reports suggest that heterophile antibody tests may be positive in Lyme disease. ${ }^{1,3-5}$ No prior cases of concurrent acute Lyme and EBV infections have been reported. We describe the clinical presentation of two children with confirmed early Lyme disease who also had clinical and laboratory features suggestive of infectious mononucleosis.

\section{Case I}

During summer, a previously healthy 5 -year-old boy living in a Lyme endemic area presented to his pediatrician with a seven day history of abdominal pain, intermittent tactile fevers, neck pain, fatigue, and sore throat. Examination revealed anterior cervical lymphadenopathy and throat

Corresponding Author: Holly M. Frost, MD, Denver Health Medical Center, University of Colorado School of Medicine, 777 Bannock Street, Denver, CO 80204, Email: holly.frost2@dhha.org erythema, but no rash or hepatosplenomegaly. The patient had no recent history of tick bite. Complete blood count (CBC) showed normal white blood cell count (WBC) of $8 \mathrm{x}$ $10^{3} / \mu \mathrm{L}$ white blood cells with no atypical lymphocytes. Heterophile antibody testing was positive. EBV serology was not obtained. Lyme studies, including the enzyme immunoassay (EIA) screening test and IgM immunoblot, (bands 23, 39, $41 \mathrm{kDa}$ ) were positive. Provider notes indicated that Lyme positivity was thought to be secondary to crossreactive antibodies and supportive care was recommended. Two days later the patient returned with multiple erythema migrans indicative of early disseminated Lyme disease. He was started on 14 days of amoxicillin and had near-complete resolution of symptoms after four days of treatment with stomach pain as the only remaining symptom.

\section{Case 2}

In spring, a previously healthy 8 -year-old boy living in a Lyme endemic region developed fever $\left(40^{\circ} \mathrm{C}\right)$, headache, sore throat, abdominal pain, fatigue, myalgia, and joint pain starting in the right elbow and progressing to the left wrist and ankle. Throat erythema and cervical lymphadenopathy were present on exam. He had no rash or history of recent tick bite. Laboratory testing was negative for Group A Streptococcus pharyngitis and heterophile antibodies. CBC had a normal $\mathrm{WBC}$ of $11.0 \times 10^{3} / \mu \mathrm{L}$ with $7 \%$ atypical

Accepted: July 17, 2018 


\section{A. Day 4 of Illness}

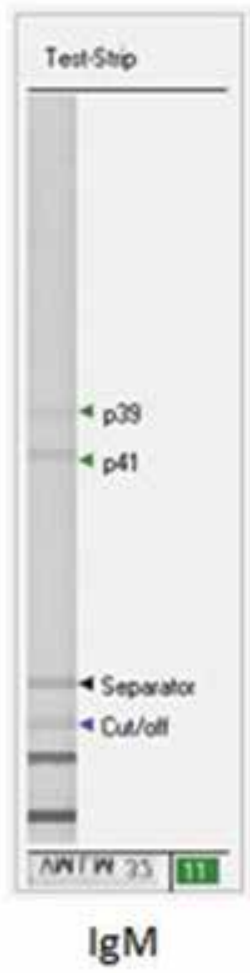

\section{B. Day 11 of Illness}

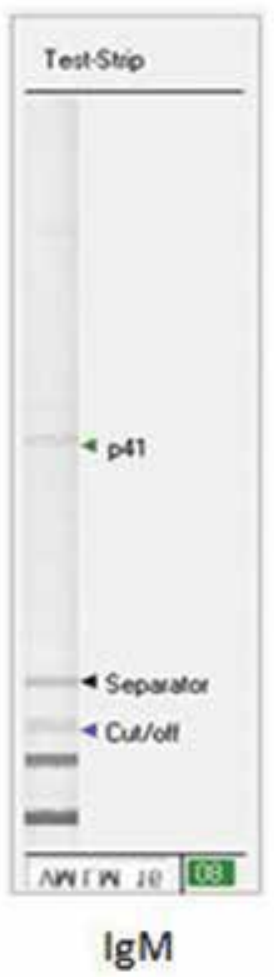

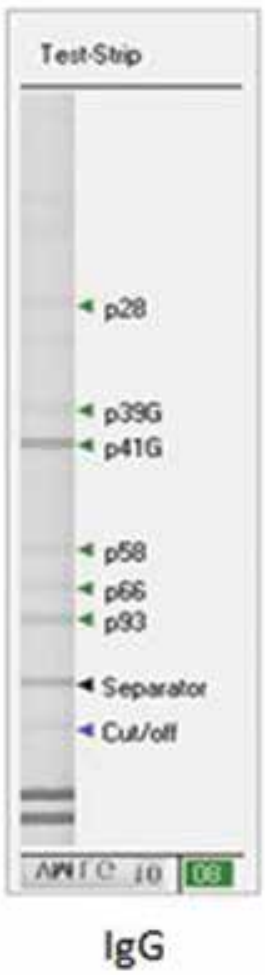

Figure 1. Lyme $\lg M$ and $\lg G$ immunoblots for Case 2 obtained at $(A)$ day 4 and (B) day 11 of illness.

lymphocytes, CRP was $0.5(\mathrm{mg} / \mathrm{dL})$, and ESR was $33(\mathrm{~mm} /$ hr). Lyme screening EIA and IgM immunoblot (bands 39, 41 $\mathrm{kDa}$ ) were positive. Polymerase chain reaction (PCR) testing for anaplasmosis, erhlichiosis, and babesiosis was negative. The patient was started on amoxicillin, but returned to clinic one week later with continued fevers and worsening abdominal and joint pain. Exam and abdominal ultrasound confirmed splenomegaly and lymphadenopathy, but no hepatomegaly. Liver enzymes were not assessed. EBV serology returned positive for VCA IgM and EA-D IgG antibodies, and repeat Lyme serology showed evidence for sero-conversion (bands 28, 39, 41, 58, 66, $93 \mathrm{kDa}$ ) (Figure 1). He completed 21 days of amoxicillin with a complete recovery.

\section{Discussion}

Lyme disease was confirmed in both cases by erythema migrans or seroconversion. Some clinical features including sore throat, cervical lymphadenopathy and splenomegaly (Case 2) were more consistent with infectious mononucleosis than Lyme disease. EBV serology and heterophile antibody results presented a diagnostic challenge in both cases. In Case 1 , the heterophile antibody tests results and the known crossreactivity of Lyme antibody assays led the provider to conclude that the Lyme results were a false positive and thus, delayed initiation of antibiotics. The absence of atypical lymphocytes and abrupt resolution of symptoms with antibiotic initiation raised suspicion for a false positive heterophile antibody test in this case, though concurrent infection could not be excluded. Previous case reports have described similar scenarios to Case 1, often with patients presenting with early disseminated Lyme disease that is misdiagnosed as infectious mononucleosis secondary to a false positive heterophile antibody or EBV VCA-IgM test. ${ }^{1,3}$ Our patient was fortunate in that he did not develop severe sequelae. In two prior reports severe disease including carditis and brachial plexopathy developed after the misdiagnosis and delay in antibiotic initiation. ${ }^{1,3}$

In Case 2, the presence of atypical lymphocytes combined with EBV VCA-IgM, and ultrasound-confirmed splenomegaly, suggest concurrent Lyme and EBV infection. To our knowledge, this is the first case of probable EBV and Lyme disease co-infection reported. Of note, neither patient developed rash after initiation of amoxicillin; however, this is thought to occur in fewer than $30 \%$ of patients with infectious mononucleosis who take amoxicillin. ${ }^{6}$

EBV serology should be highly sensitivity for mononucleosis; however, cross-reactivity between EBV VCA and Lyme IgM can occur. ${ }^{3,4}$ Numerous studies have demonstrated false positive Lyme IgM in the setting of EBV infection, while only two cases of false positive EBV IgM in the setting of acute Lyme disease have been published. ${ }^{3,4}$ As a result, positive Lyme serology is sometimes dismissed as cross-reactivity in patients with symptoms of infectious mononucleosis and EBV 
VCA IgM antibodies, which can delay diagnosis and treatment for Lyme disease.

Heterophile antibody tests have lower sensitivity than EBV serology for infectious mononucleosis, particularly in children less than age 4 years. ${ }^{5}$ Heterophile antibody tests have specificities of $96 \%$ to $100 \%$ for infectious mononucleosis. ${ }^{5}$ However, false positive heterophile antibody tests have been reported in patients with rubella, malaria, malignancy, rheumatoid arthritis, as well as two patients with Lyme disease. ${ }^{1,5}$ The etiology of false positive heterophile antibody tests is unclear, though non-specific production of heterophile antibodies in severe infections are thought to play a role. ${ }^{1}$ Concurrent evaluation for EBV VCA IgM, heterophile antibodies, and atypical lymphocytes in the acute setting is likely to improve specificity. ${ }^{5}$ Ultimately, acute and convalescent EBV and Lyme serology to assess for seroconversion is the most accurate method to confirm a diagnosis. However, providers should be aware that seroconversion may not occur in patients treated for Lyme early in the disease course. Given the retrospective nature of this study we were unable to look for EBV seroconversion in these cases, which limits the ability to confirm active EBV infection. However, the presence of sore throat, severe cervical lymphadenopathy, ultrasound confirmed splenomegaly, and atypical lymphocytes in Case 2 is highly suggestive of active EBV infection.

Though false positive EBV serology in the presence of active Lyme disease has been reported, these cases did not present the possibility of concurrent infection, as we report here. ${ }^{1,3}$ The possibility of concurrent Lyme and EBV infection is not surprising as both infections are common in children in Lyme endemic regions. ${ }^{1,7}$ In a retrospective review of laboratory data at Marshfield Clinic Health System from 1999-2017 we identified 52 patients with positive Lyme $\operatorname{IgM}$ along with positive heterophile antibody test or EBV VCA IgM within a 2 week window. It is possible that EBV or Lyme infection renders the host more susceptible to co-infection, particularly in the setting of treatment with corticosteroids for EBV; however, this has not been studied.

Over $95 \%$ of adults world-wide have serologic evidence of prior EBV infection. ${ }^{1}$ EBV re-activation is known to occur in times of immunocompromised state, cellular stress, and inflammation. ${ }^{8}$ Though unlikely in the cases presented, it is interesting to consider whether the induced stress of Lyme disease is capable of re-activating EBV. Little is known regarding this topic, however, it has been shown that when EBV infected lymphoblastoid cells are superinfected with Borrelia garinii, a Lyme inducing spirochete found in Eurasia, the infected cells displayed accelerated EBV replication. ${ }^{8}$

In summary, clinicians in Lyme endemic areas should maintain a high index of suspicion for Lyme disease in children presenting with acute febrile illness. Heterophile antibody testing and EBV serology should not be used to exclude the diagnosis of Lyme disease or delay treatment. Testing of acute and convalescent serum in patients with concern for EBV or Lyme disease can confirm the diagnosis in ambiguous cases.

\section{Acknowledgements}

We would like to thank Matt Hall, MD for providing his clinical expertise and reviewing the manuscript.

\section{References}

1. Tumminello R, Glaspey L, Bhamidipati A, Sheehan P, Patel S. Early Disseminated Lyme Disease Masquerading as Mononucleosis: A Case Report. J Emerg Med 2017;53(6):e133-e135.

2. Lipsett SC, Nigrovic LE. Diagnosis of Lyme disease in the pediatric acute care setting. Curr Opin Pediatr 2016;28(3):287-293.

3. Pavletic AJ, Marques AR. Early Disseminated Lyme Disease Causing False-Positive Serology for Primary Epstein-Barr Virus Infection: Report of 2 Cases. Clin Infect Dis 2017;65(2):336-337.

4. Seriburi V, Ndukwe N, Chang Z, Cox ME, Wormser GP. High frequency of false positive IgM immunoblots for Borrelia burgdorferi in clinical practice. Clin Microbiol Infect 2012;18(12):1236-1240.

5. Marshall-Andon T, Heinz P. How to use ... the Monospot and other heterophile antibody tests. Arch Dis Child Educ Pract Ed. 2017;102(4):188-193.

6. Chovel-Sella A, Ben Tov A, Lahav E, et al. Incidence of rash after amoxicillin treatment in children with infectious mononucleosis. Pediatrics 2013;131(5):e1424-e1427.

7. Onyett H; Canadian Paediatric Society, Infectious Diseases and Immunization Committee. Lyme disease in Canada: Focus on children. Paediatr Child Health. 2014;19(7):379-388.

8. Hulínská D, Roubalová K, Schramlová J. Interaction of Borrelia burgdorferi sensu lato with Epstein-Barr virus in lymphoblastoid cells. Folia Biol (Praha). 2003;49(1):40-48.

\section{Author Affiliations}

Trever M. Koester, BS*,†; Jennifer K. Meece, PhD†; Thomas

R. Fritsche, MD $\$$; and Holly M. Frost, MD†,\|ף

*University of Wisconsin-Stout, Menomonie, WI, USA

†Integrated Research and Development Laboratory, Marshfield Clinic Research Institute, Marshfield, WI, USA

$¥$ Division of Laboratory Medicine, Marshfield Clinic Health System, Marshfield, WI, USA

$\S$ Department of Microbiology, University of Wisconsin-La

Crosse, La Crosse, WI, USA

$\|$ Department of Pediatrics, Denver Health Medical Center,

Denver, CO, USA

-Department of Pediatrics, University of Colorado School of Medicine, Denver, CO, USA 